\title{
Psychological Impacts of the COVID-19 Pandemic on the Public in Egypt
}

\author{
Ahmed Arafa ${ }^{1,2,4}\left(\mathbb{C} \cdot\right.$ Amel Mohamed $^{3} \cdot$ Lamiaa Saleh $^{2} \cdot$ Shaimaa Senosy $^{2}$
}

Received: 16 May 2020 / Accepted: 12 August 2020 / Published online: 16 August 2020

(c) Springer Science+Business Media, LLC, part of Springer Nature 2020

\begin{abstract}
This study aimed to evaluate the prevalence of depression, anxiety, stress, and inadequate sleeping among the public in Egypt during the novel Corona Virus Disease (COVID-19) pandemic. An online snowball sampling approach was used to collect data from netizens in four Egyptian governorates between 16 and 30 April 2020. A semi-structured questionnaire was designed to assess sociodemographic characteristics, sleeping hours per day, and psychological disturbances (depression, anxiety, and stress) of participants using the Depression Anxiety Stress Scale-21. Out of 1629 participants, $48.1 \%$ were aged $\leq 30$ years, $42.4 \%$ were men, and $20.0 \%$ were working in the health sector. The participants reported a high prevalence of depression (67.1\%: mild to moderate $44.6 \%$ and severe to very severe $22.5 \%$ ), anxiety $(53.5 \%$ : mild to moderate $30.6 \%$ and severe to very severe $22.9 \%$ ), stress $(48.8 \%$ : mild to moderate $33.8 \%$ and severe to very severe $15.0 \%$ ), and inadequate sleeping ( $<6 \mathrm{~h} /$ day) $23.1 \%$. Female sex, working in sectors other than the health sector, watching/reading COVID-19 news $\geq 2 \mathrm{~h} /$ day, and lack of emotional support from family and society were associated with a high prevalence of severe to very severe depression, anxiety, and stress. In conclusion, the psychological impacts of COVID-19 on the public in Egypt were enormous, therefore, providing psychological support and counsel is warranted.
\end{abstract}

Keywords COVID-19 · Depression · Anxiety · Stress · Inadequate sleeping

\section{Abbreviations \\ CI Confidence interval \\ COVID-19 The novel Corona Virus Disease-2019 \\ DASS-21 Depression Anxiety Stress Scale-21 \\ OR Odds ratio}

Ahmed Arafa

ahmed011172@med.bsu.edu.eg;

dr_ahmed_emad_arafa@yahoo.com

Lamiaa Saleh

lamiaasaleh@yahoo.com

Shaimaa Senosy

shoshoahmed80@yahoo.com

1 Department of Public Health, Graduate School of Medicine, Osaka University, Osaka, Japan

2 Department of Public Health, Faculty of Medicine, Beni-Suef University, Beni-Suef, Egypt

3 Department of Community Health, Faculty of Nursing, Beni-Suef University, Beni-Suef, Egypt

4 Public Health, Department of Social Medicine, Osaka University Graduate School of Medicine, 2-2 Yamadaoka, Suita, Osaka 565-0871, Japan
SPSS Statistical Package for Social Science

WHO World Health Organization

\section{Introduction}

The evolution of the novel Corona Virus Disease (COVID19) and its expeditious dissemination have led the World Health Organization (WHO) to declare it a pandemic on 11 March 2020 (WHO 2020a). As of 16 May 2020, there have been 4,425,485 confirmed COVID-19 cases and 302,059 related deaths worldwide and Egypt has been among the most affected countries in the Eastern Mediterranean region with 11,228 confirmed cases and 592 deaths (WHO 2020b).

As a consequence, many countries, including Egypt, have set a state of lockdown to hinder the spread of infection among people resulting in a global atmosphere of depression and anxiety associated with social isolation, fears of getting infected, breaks in the supply chains, financial distress, disrupted travel plans, and future uncertainty (Hiremath et al. 2020; Ho et al. 2020; Zhang et al. 2020). Several epidemiological studies from China detected a high prevalence of psychological disturbances among the public during the 
COVID-19 pandemic. For example, a large online survey of 56,679 individuals from 34 provinces in China reported numerous psychological conditions during the COVID19 pandemic: depression (27.9\%), anxiety (31.6\%), stress (24.4\%), and insomnia (29.2\%) (Shi et al. 2020). Also, a study conducted on 4872 Chinese people from 31 provinces showed a high prevalence of depression (48.3\%) and anxiety (22.6\%) related to the COVID-19 pandemic (Gao et al. 2020). A similar study revealed depression $(20.1 \%)$, anxiety (35.1\%), and poor sleeping (18.2\%) among 7236 Chinese people (Huang and Zhao 2020). Another study conducted on 7143 college students in China detected anxiety among $25 \%$ of them (Cao et al. 2020).

In low- and middle-income countries, such as Egypt, where infection control precautions, surveillance programs, laboratory capacity, and public health resources are limited (Asbu et al. 2017), the response to the COVID-19 pandemic is challenging and, as a result, the psychological impacts of the pandemic on the public can be even worse. Thus, we conducted this cross-sectional study to evaluate several forms of psychological disturbances (depression, anxiety, stress, and inadequate sleeping) and their associating factors among the public in four governorates in Egypt during the COVID-19 pandemic. We hope that the findings of this study can help in spotting the population at high risk of psychological disturbances in Egypt and detecting potential associations for possible psychological interventions during this pandemic or future waves of infection.

\section{Methods}

\section{Study Design, Population, and Setting}

As per the Egyptian Government guidelines to the public for reducing face-to-face communication and home isolation, we used an online snowball sampling procedure to collect data from people aged $\geq 18$ years old and residing in Egypt during the period between 16 and 30 April 2020 .

The lowest sample size was calculated using the EpiInfo version $7 \mathrm{StatCalc}$, which is available from the Centers for Disease Control (CDC) and the WHO. After reviewing previous studies, we determined the following criteria to calculate the least sample size: population size of 999,999, expected frequency of $50 \%$, confidence level of $95 \%$, and a margin of error of $5 \%$. However, we then more than quadrupled the least sample size to serve in stratifying statistical analyses of depression, anxiety, and stress into mild to moderate and severe to very severe forms and to overcome any unexpected invalid questionnaires.

Four Egyptian governorates: Alexandria and Cairo in northern Egypt and Beni-Suef and Assiut in southern Egypt were nonrandomly selected for data collection. Cairo is the
Capital of Egypt and the most populous governorate in the country while Assiut is the most populous governorate in southern Egypt. Beni-Suef is one of the most underprivileged governorates in the country with major deficits in health and educational services whereas Alexandria represents the country's modern face with better health and educational services (CAPMAS 2017).

We designed a semi-structured questionnaire using Google forms, then, we shared the link to the questionnaire to numerous Facebook groups hosting netizens from the four governorates. Facebook is the most widespread social network among the Egyptian netizens (Arafa et al. 2019a). We used the names of the four governorates as search terms to select the appropriate Facebook groups for sharing our link. Respondents were asked to forward the questionnaire to their eligible friends in their social network contact lists. We managed to collect 425 questionnaires from each governorate; therefore, data collection was terminated in the governorates that reached the required sample before the due date of 30 April 2020 (Beni-Suef: 28 April, Assiut: 29 April, Cairo: 29 April, and Alexandria 30 April). Eventually, 1700 questionnaires were obtained from the four governorates before 71 questionnaires were excluded for being invalid questionnaires or the respondents reported staying outside Egypt during the time of data collection.

\section{Data Collecting Tool}

The questionnaire was composed of two sections. The first section included questions about sociodemographic characteristics including age (18-30,31-35, or $>45$ years), sex (man or woman), governorate (Alexandria, Cairo, BeniSuef, or Assiut), current work sector (health, education, services, instruction, trading, industry, other sectors, or none), education (elementary or high), living with children (yes or no) or old people (yes or no), watching or reading news about the pandemic $(<2,2-4$, or $>4 \mathrm{~h} /$ day $)$, receiving enough emotional support from family (yes or no) and society (yes or no) during the pandemic, and sleeping hours/ day during the past month ( $\geq 6$ or $<6 \mathrm{~h} /$ day). The second section included the Arabic version of the Depression Anxiety Stress Scale-21 (DASS-21). Based on 21 statements, people were asked to decide on how close the DASS-21 statements expressed their status during the previous week on a four-point scale between zero and three with higher scores indicating worse psychological status. The depression statements assessed hopelessness, dysphoria, self-deprecation, devaluation of life, lack of interest, anhedonia, and inertia. The anxiety statements evaluated skeletal muscle effects, autonomic arousal, situational anxiety, and subjective experience of anxious affect. The stress scale evaluated nervous arousal, difficulties in relaxation, and being easily upset or over-reactive. Scores of statements under the same 
emotional category (seven statements each) were summed and multiplied in two. Out of 42 , the cut-off values for mild to moderate depression, anxiety, and stress were 10,8 , and 15 , and for severe to very severe were 22,16 , and 26 , respectively (Lovibond and Lovibond 1995). The Cronbach's $\alpha$ for the Arabic version of DASS-21 was 0.88 (Ali et al. 2017). In this study, depression, anxiety, stress, and sleeping $<6 \mathrm{~h} /$ day (referred to as inadequate sleeping) were considered outcomes.

\section{Ethical Consideration}

Expedited ethics approval was received from the Research Ethics Committee of the Faculty of Medicine, Beni-Suef University which adhered to the principles of the Declaration of Helsinki. All respondents replied to a question if they agree to participate in this study before filling out the questionnaire.

\section{Statistical Analyses}

Data were analyzed with the Statistical Package for Social Science (SPSS). Frequency measures were used to assess the prevalence of depression, anxiety, stress, and inadequate sleeping among the public. The logistic regression analyses were used to compute the odds ratios (ORs) and 95\% confidence intervals (CIs) of different sociodemographic factors for people with severe to very severe depression, anxiety, and stress and inadequate sleep compared with people without the corresponding psychological conditions. The following covariates were included in the regression model: age, sex, governorate, profession, education, living with children and older adults, watching/reading COVID-19 news, and getting emotional support from family and society.

\section{Results}

This study included 1629 people (25.0\% from Alexandria, $24.5 \%$ from Cairo, $25.1 \%$ from Beni-Suef, and $25.4 \%$ from Assiut). Of them, $48.1 \%$ were aged $\leq 30$ years, $42.4 \%$ were men, and 20\% were working in the health sector (Table 1).

The results revealed a high prevalence of depression (67.1\%: mild to moderate $44.6 \%$ and severe to very severe $22.5 \%$ ), anxiety (53.5\%: mild to moderate $30.6 \%$ and severe to very severe $22.9 \%$ ), stress $(48.8 \%$ : mild to moderate $33.8 \%$ and severe to very severe $15.0 \%$ ), and inadequate sleeping $23.1 \%$ (Table 2).

In the multivariable-adjusted regression model, female sex, working in sectors other than the health sector, watching/reading COVID-19 news $\geq 2 \mathrm{~h} /$ day, and lack of emotional support from family and society associated with a high prevalence of psychological disturbances. Compared with men, women
Table 1 Sociodemographic characteristics of the participants

\begin{tabular}{|c|c|}
\hline Characteristics & $\begin{array}{l}\text { Overall } \\
\mathrm{n}=1629(\%)\end{array}$ \\
\hline \multicolumn{2}{|l|}{ Age (years) } \\
\hline $18-30$ & $784(48.1)$ \\
\hline $31-35$ & $470(28.9)$ \\
\hline$>45$ & $375(23.0)$ \\
\hline \multicolumn{2}{|l|}{ Sex } \\
\hline Men & $690(42.4)$ \\
\hline Women & 939 (57.6) \\
\hline \multicolumn{2}{|l|}{ Governorate } \\
\hline Alexandria & $407(25.0)$ \\
\hline Cairo & $399(24.5)$ \\
\hline Beni-Suef & $409(25.1)$ \\
\hline Assiut & $414(25.4)$ \\
\hline \multicolumn{2}{|l|}{ Profession (sector) } \\
\hline Health & $326(20.0)$ \\
\hline Education & $273(16.8)$ \\
\hline Services & $169(10.4)$ \\
\hline Instruction & $161(9.9)$ \\
\hline Trading & $153(9.4)$ \\
\hline Industry & $136(8.3)$ \\
\hline Others & $101(6.2)$ \\
\hline None & $310(19.0)$ \\
\hline \multicolumn{2}{|l|}{ Education } \\
\hline Elementary & $320(19.6)$ \\
\hline High & $1309(80.4)$ \\
\hline Living with children & $1172(71.9)$ \\
\hline Living with older adults & $905(55.6)$ \\
\hline Emotional support from family & $1228(75.4)$ \\
\hline Emotional support from society & $822(50.5)$ \\
\hline \multicolumn{2}{|c|}{ Watching/reading COVID-19 news (h/day) } \\
\hline$<2$ & $981(60.2)$ \\
\hline $2-4$ & $446(27.4)$ \\
\hline$>4$ & $202(12.4)$ \\
\hline
\end{tabular}

reported more severe to very severe depression (OR 1.39, $95 \%$ CI $1.09,1.78$ ), anxiety (OR $2.64,95 \%$ CI $1.85,3.29$ ), and stress (OR 2.90, 95\% CI 2.04, 4.11). Watching/reading COVID-19 news $\geq 2 \mathrm{~h} /$ day was associated with severe to very severe depression (OR 3.60, 95\% CI 2.64, 4.90), anxiety (OR $3.16,95 \%$ CI 2.39, 4.19), and stress (OR 2.58, 95\% CI 1.88, 3.54). Lack of emotional support from family and society was also associated with severe to very severe depression, anxiety, and stress (Table 3).

\section{Discussion}

This study is one of the earliest studies to evaluate the prevalence of several psychological disturbances among the public outside China during the COVID-19 pandemic. We 
Table 2 Prevalence of depression, anxiety, stress, and inadequate sleeping among the public during the COVID-19 pandemic

\begin{tabular}{lc}
\hline Psychological features & $\begin{array}{l}\text { Overall } \\
\mathrm{n}=1629(\%)\end{array}$ \\
\hline Depression & $536(32.9)$ \\
Normal & $726(44.6)$ \\
Mild to moderate & $367(22.5)$ \\
Severe to very severe & \\
Anxiety & $758(46.5)$ \\
Normal & $499(30.6)$ \\
Mild to moderate & $372(22.9)$ \\
Severe to very severe & \\
Stress & $835(51.2)$ \\
Normal & $550(33.8)$ \\
Mild to moderate & $244(15.0)$ \\
Severe to very severe & \\
Sleeping (h/day) & $1252(76.9)$ \\
$\geq 6$ & $377(23.1)$ \\
\hline 66 &
\end{tabular}

indicated a high prevalence of depression (67.1\%), anxiety (53.5\%), stress (48.8\%), and inadequate sleeping (23.1\%) among the public in Egypt during the pandemic. We could also determine many sociodemographic associations with severe to very severe forms of depression, anxiety, and stress.

It should be noted that comparing our results with the previous Chinese studies could be challenging due to the wide differences in sociodemographic characteristics and cultural perspectives between both populations in addition to the variations in the scales assessing psychological conditions across studies. However, we could notice that the prevalence of psychological conditions among our sample was higher than that among the Chinese populations. This could be explained by the limited health services in Egypt in comparison with China which led to worse psychological conditions. This explanation is supported by our findings that showed more severe to very severe depression and anxiety among southern governorates (Beni-Suef and Assiut) than northern governorates (Cairo and Alexandria). The health system in southern Egypt is inferior to that in northern Egypt with many financial, structural, and cultural barriers to health services in the southern governorates (Chiang et al. 2013). Moreover, the lower income, that characterizes the Egyptian population compared with the Chinese one and the southern Egyptians compared with the northern ones, was shown to augment the psychological conditions during the COVID-19 pandemic (Shi et al. 2020; Cao et al. 2020).

Surprisingly, people working in non-health sectors reported more psychological disturbances than people working in the health sector. In line with our findings, a meta-analysis of studies assessing the psychological conditions among healthcare workers during the COVID-19 pandemic showed that the prevalence of depression was 5.1\% and $25.5 \%$ lower than two large Chinese studies conducted on the general population (Pappa et al. 2020; Shi et al. 2020; Gao et al. 2020). This could be partially explained by the possible better knowledge and more positive attitude towards the pandemic among healthcare workers compared with other occupations, therefore, they would be more able to develop coping strategies with the pandemic. In contrast, a web-based cross-sectional survey from China showed that healthcare workers were more likely to suffer mental health problems than the public during the COVID-19 pandemic (Huang and Zhao 2020).

Our results showed that women were more likely to suffer psychological disturbances than men which reflects the already known gender gap for depression and anxiety (Albert 2015). In agreement, pooling the results of five recent studies showed a depression prevalence of $20.3 \%$ among men and $26.9 \%$ among women. Also, pooling the results of six studies showed an anxiety prevalence of $20.9 \%$ among men and $29.1 \%$ among women (Pappa et al. 2020).

Further, watching/reading COVID-19 news $\geq 2$ h/day, in this study, was associated with depression, anxiety, and stress. Exposure to social media and thinking about the pandemic for a long time were associated with worse psychological consequences (Gao et al. 2020; Huang and Zhao 2020). Dong and Zheng (2020) recently described what they referred to as "headline stress disorder" among 1206 general public in China due to news related to the COVID-19 pandemic. Alike, media coverage of the Ebola outbreak was associated with anxiety among the public in Africa (Kilgo et al. 2019).

Moreover, people who lacked emotional support from family and society showed worse psychological consequences compared with their counterparts who were offered support. A study conducted on college students in China showed that social support correlated negatively with their level of anxiety during the COVID-19 pandemic (Cao et al. 2020). Greater attention, therefore, should be paid to the public mental health by actively providing them with psychological support and counsel.

Although this study evaluated the psychological impacts of the COVID-19 pandemic on a large sample of netizens from northern and southern Egypt using a validated assessment tool, some limitations should be considered. First, because of the cross-sectional design of the study, a causality association cannot be implied. Second, the possibility of non-response bias remains a major shortcoming of online questionnaires (Arafa et al. 2019b). To minimize the effect of this bias, the questionnaire did not involve sensitive questions and used simple multi-choice questions. Still, we cannot guarantee the representativeness of 
Table 3 Sociodemographic associations with severe to very severe depression, anxiety, and stress and inadequate sleeping

\begin{tabular}{|c|c|c|c|c|c|c|c|c|}
\hline \multirow[t]{2}{*}{ Characteristics } & \multicolumn{2}{|c|}{$\begin{array}{l}\text { Severe to very severe depres- } \\
\text { sion }\end{array}$} & \multicolumn{2}{|c|}{ Severe to very severe anxiety } & \multicolumn{2}{|c|}{ Severe to very severe stress } & \multicolumn{2}{|c|}{$\begin{array}{l}\text { Inadequate sleeping } \\
(<6 \mathrm{~h} / \text { day })\end{array}$} \\
\hline & $\%$ & OR $(95 \% \mathrm{CI})$ & $\%$ & OR $(95 \%$ CI $)$ & $\%$ & OR $(95 \%$ CI $)$ & $\%$ & OR $(95 \%$ CI $)$ \\
\hline \multicolumn{9}{|l|}{ Age } \\
\hline$<30$ years & 24.0 & $1.08(0.78,1.49)$ & 21.0 & $0.76(0.56,1.03)$ & 14.9 & $0.93(0.66,1.31)$ & 16.3 & $0.49(0.37,0.64)$ \\
\hline$\geq 30$ years & 21.2 & 1 & 24.5 & 1 & 15.0 & 1 & 29.5 & 1 \\
\hline \multicolumn{9}{|l|}{ Sex } \\
\hline Women & 26.6 & $2.13(1.57,2.91)$ & 27.4 & $2.64(1.85,3.29)$ & 20.0 & $2.90(2.04,4.11)$ & 22.0 & $0.82(0.64,1.04)$ \\
\hline Men & 17.0 & 1 & 16.7 & 1 & 8.1 & 1 & 24.6 & 1 \\
\hline \multicolumn{9}{|l|}{ Governorate } \\
\hline Alexandria & 19.7 & $0.56(0.38,0.85)$ & 17.9 & $0.47(0.32,0.69)$ & 13.5 & $0.83(0.53,1.30)$ & 23.3 & $1.03(0.73,1.44)$ \\
\hline Cairo & 19.8 & $0.71(0.45,1.11)$ & 19.0 & $0.47(0.31,0.72)$ & 16.5 & $1.04(0.65,1.66)$ & 28.8 & $1.27(0.90,1.80)$ \\
\hline Beni-Suef & 22.7 & $0.90(0.59,1.36)$ & 25.9 & $0.84(0.58,1.21)$ & 16.4 & $1.08(0.69,1.69)$ & 18.6 & $0.88(0.62,1.25)$ \\
\hline Assiut & 27.8 & 1 & 28.3 & 1 & 13.5 & 1 & 22.0 & 1 \\
\hline \multicolumn{9}{|l|}{ Profession } \\
\hline Health sector & 19.3 & $0.56(0.38,0.81)$ & 18.4 & $0.60(0.42,0.85)$ & 16.0 & $0.73(0.50,1.06)$ & 22.1 & $1.07(0.79,1.46)$ \\
\hline Other sectors* & 23.3 & 1 & 23.9 & 1 & 14.7 & 1 & 23.4 & 1 \\
\hline \multicolumn{9}{|l|}{ Education } \\
\hline Elementary & 19.1 & $1.01(0.81,1.47)$ & 27.2 & $1.82(1.28,2.60)$ & 9.1 & $0.58(0.37,0.93)$ & 29.1 & $1.39(1.04,1.87)$ \\
\hline High & 23.4 & 1 & 21.8 & 1 & 16.4 & 1 & 21.7 & 1 \\
\hline \multicolumn{9}{|c|}{ Living with children } \\
\hline Yes & 21.2 & $0.90(0.65,1.24)$ & 23.3 & $1.21(0.89,1.65)$ & 15.4 & $1.10(0.78,1.57)$ & 23.8 & $1.00(0.76,1.32)$ \\
\hline No & 25.8 & 1 & 21.7 & 1 & 13.8 & 1 & 21.4 & 1 \\
\hline \multicolumn{9}{|c|}{ Living with older adults } \\
\hline Yes & 23.1 & $1.09(0.81,1.47)$ & 23.1 & $1.15(0.87,1.51)$ & 15.2 & $1.06(0.77,1.45)$ & 23.5 & $1.20(0.94,1.53)$ \\
\hline No & 21.8 & 1 & 22.5 & 1 & 14.6 & 1 & 22.7 & 1 \\
\hline \multicolumn{9}{|c|}{ Watching/reading COVID-19 news (h/day) } \\
\hline$\geq 2$ & 30.1 & $3.60(2.64,4.90)$ & 32.3 & $3.16(2.39,4.19)$ & 19.0 & $2.58(1.88,3.54)$ & 23.0 & $0.87(0.68,1.11)$ \\
\hline$<2$ & 17.5 & 1 & 16.6 & 1 & 12.3 & 1 & 23.2 & 1 \\
\hline \multicolumn{9}{|c|}{ Getting emotional support from family } \\
\hline No & 40.1 & $3.97(2.76,5.70)$ & 34.4 & $2.34(1.70,3.23)$ & 25.7 & $2.42(1.72,3.42)$ & 26.4 & $1.29(0.97,1.72)$ \\
\hline Yes & 16.8 & 1 & 19.1 & 1 & 11.5 & 1 & 22.1 & 1 \\
\hline \multicolumn{9}{|c|}{ Getting emotional support from society } \\
\hline No & 31.6 & $3.08(2.23,4.23)$ & 28.9 & $2.18(1.62,292)$ & 21.2 & $2.93(2.08,4.12)$ & 22.7 & $0.97(0.75,1.25)$ \\
\hline Yes & 13.6 & 1 & 16.9 & 1 & 8.9 & 1 & 23.6 & 1 \\
\hline
\end{tabular}

Adjusted for age, sex, governorate, profession, education, living with children and older adults, watching/reading COVID-19 news, and emotional support from family and society

*Including the unemployed

the study population because all respondents were internet users who could read and write. Egypt suffers high illiteracy rates and illiterate people are significantly different from the educated people regarding their sociodemographic characteristics (CAPMAS 2017). Third, some potential confounders that can modify the mental health impacts of the pandemic such as leisure activities were not investigated. Fourth, receiving emotional support from family and society was subjectively assessed using two yes/no questions. Using more detailed scales to assess emotional support would have been more informative.

In conclusion, the psychological impacts of the COVID19 pandemic on the public were enormous, especially among women and people not working in the health sector, therefore, providing psychological support to the public is highly desirable. 
Author Contributions Design: AA and SS, data collection: AM, LS, and SS, statistical analysis: AA, and writing the manuscript and serious revision: AA, AM, LS, and SS.

Funding None.

\section{Compliance with Ethical Standards}

Conflict of interest The authors declare no conflict of interest.

\section{References}

Albert, P. (2015). Why is depression more prevalent in women? Journal of Psychiatry \& Neuroscience, 40, 219-221.

Ali, A., Ahmed, A., Sharaf, A., Kawakami, N., Abdeldayem, S., \& Green, J. (2017). The Arabic version of the Depression Anxiety Stress Scale-21: Cumulative scaling and discriminant-validation testing. Asian Journal of Psychiatry, 30, 56-58.

Arafa, A., Saif, S. A., Ramadan, A., Rashed, T., Ahmed, S., \& Taha, M. (2019a). Problematic internet use: A cross-sectional study on a model from university students in Egypt. International Journal of Adolescent Medicine and Health. https://doi.org/10.1515/ijamh -2019-0031.

Arafa, A., Anzengruber, F., Mostafa, A., \& Navarini, A. (2019b). Perspectives of online surveys in dermatology. Journal of the European Academy of Dermatology and Venereology, 33, 511-520.

Asbu, E., Masri, M., \& Kaissi, A. (2017). Health status and health systems financing in the MENA region: Roadmap to universal health coverage. Global Health Research Policy, 2, 25.

Cao, W., Fang, Z., Hou, G., Han, M., Xu, X., Dong, J., et al. (2020). The psychological impact of the COVID-19 epidemic on college students in China. Psychiatry Research, 287, 112934.

Central Agency for Public Mobilization and Statistics (CAPMAS). (2017). Egypt statistics. Final results of 2017 Census. https:// www.capmas.gov.eg. Accessed on 4 August 2020.

Chiang, C., Labeeb, S. A., Higuchi, M., Mohamed, A. G., \& Aoyama, A. (2013). Barriers to the use of basic health services among women in rural southern Egypt (Upper Egypt). Nagoya Journal of Medical Science, 75(3-4), 225-231.

Dong, M., \& Zheng, J. (2020). Letter to the editor: Headline stress disorder caused by Netnews during the outbreak of COVID-19. Health Expectations, 23, 259-260.

Gao, J., Zheng, P., Jia, Y., Chen, H., Mao, Y., Chen, S., et al. (2020). Mental health problems and social media exposure during COVID-19 outbreak. PLoS One, 15, e0231924.
Hiremath, P., Suhas Kowshik, C., Manjunath, M., \& Shettar, M. (2020). COVID 19: Impact of lock-down on mental health and tips to overcome. Asian Journal of Psychiatry, 51, 102088.

Ho, C., Chee, C., \& Ho, R. (2020). Mental health strategies to combat the psychological impact of COVID-19 beyond paranoia and panic. Annals Academy of Medicine Singapore, 49, 1-3.

Huang, Y., \& Zhao, N. (2020). Generalized anxiety disorder, depressive symptoms and sleep quality during COVID-19 outbreak in China: A web-based cross-sectional survey. Psychiatry Research, 288, 112954.

Kilgo, D., Yoo, J., \& Johnson, T. (2019). Spreading Ebola panic: Newspaper and social media coverage of the 2014 Ebola Health Crisis. Health Communication, 34, 811-817.

Lovibond, S., \& Lovibond, P. (1995). Manual for the Depression, Anxiety and Stress Scales (2nd ed.). Sydney: Psychology Foundation.

Pappa, S., Ntella, V., Giannakas, T., Giannakoulis, V. G., Papoutsi, E., $\&$ Katsaounou, P. (2020). Prevalence of depression, anxiety, and insomnia among healthcare workers during the COVID-19 pandemic: A systematic review and meta-analysis. Brain, Behavior, and Immunity, 88, 901-907.

Shi, L., Lu, Z. A., Que, J. Y., Huang, X. L., Liu, L., Ran, M. S., et al. (2020). Prevalence of and risk factors associated with mental health symptoms among the general population in China during the Coronavirus disease 2019 pandemic. JAMA Network Open, 3(7), e2014053.

World Health Organization (WHO). (2020a). WHO Director-General's opening remarks at the media briefing on COVID-19. (Press release). 11 March 2020. https://web.archive.org/web/2020031121 2521/https:/www.who.int/dg/speeches/detail/who-director-gener al-s-opening-remarks-at-the-media-briefing-on-covid-19---11march-2020

World Health Organization (WHO). (2020b). Coronavirus disease (COVID-2019) situation reports 117. 16 May 2020. https://www. who.int/docs/default-source/coronaviruse/situation-reports/20200 516-covid-19-sitrep-117.pdf?sfvrsn=8f562cc_2.

Zhang, J., Wu, W., Zhao, X., \& Zhang, W. (2020). Recommended psychological crisis intervention response to the 2019 novel coronavirus pneumonia outbreak in China: A model of West China Hospital. Precision Clinical Medicine, 3, 3-8.

Publisher's Note Springer Nature remains neutral with regard to jurisdictional claims in published maps and institutional affiliations. 